\title{
Squared temperature-temperature power spectrum as a probe of the CMB bispectrum
}

\author{
Asantha Cooray* \\ Department of Astronomy and Astrophysics, University of Chicago, Chicago, Illinois 60637
}

(Received 17 May 2001; published 30 July 2001)

\begin{abstract}
It is now well known that mode-coupling effects associated with certain secondary effects generate higher order correlations in cosmic microwave background (CMB) temperature anisotropies, beyond the two-point function. In order to extract such a non-Gaussian signal at the three-point level, we suggest a two-point statistic in the form of an angular power spectrum involving correlations between squared temperature and temperature anisotropies. This power spectrum contains compressed information from the bispectrum and can be easily measured in data with the same techniques that have been considered for the measurement of the usual temperature-temperature anisotropy power spectrum. We study the proposed power spectrum resulting from the non-Gaussian signal generated by correlations involved with gravitational lensing angular deflections in CMB and the Sunyaev-Zel'dovich (SZ) effect due to large scale pressure fluctuations. Using the Planck frequency cleaned $\mathrm{CMB}$ and $\mathrm{SZ}$ maps, the $\mathrm{CMB}^{2}-\mathrm{SZ}$ power spectrum provides a direct estimate of the cross-power between lensing angular deflections and the SZ effect. Through an optimal filter applied to the squared CMB map, the proposed statistic allows one to obtain all information from the lensing-SZ bispectrum. The observational measurement of the lensing-SZ cross-correlation is useful to understand the relation between large scale structure pressure and dark matter fluctuations.
\end{abstract}

DOI: 10.1103/PhysRevD.64.043516

PACS number(s): 98.80.Es, 98.35.Ce, 98.70.Vc

\section{INTRODUCTION}

It is by now well accepted that precision measurements of the cosmic microwave background (CMB) expected from upcoming experiments, especially the Microwave Anisotropy Probe ${ }^{1}$ and Planck surveyor, ${ }^{2}$ will provide adequate information for a precise measurement of cosmological parameters through the $\mathrm{CMB}$ temperature anisotropy power spectrum [1]. In addition to a measurement of the angular power spectrum, these experiments provide all-sky maps across a wide range frequencies from 20 to $900 \mathrm{GHz}$, allowing the possibility to carry out a large number of secondary sciences, which are arguably as interesting as the primary goal. The possibilities for such studies with multifrequency CMB data include galactic foregrounds, secondary anisotropies such as the Sunyaev-Zel'dovich [2] effect resulting from the inverse-Compton scattering of CMB photons via hot gas in large scale structure [3] and far-infrared background due to high redshift dusty starforming galaxies [4].

The increase in sensitivity of these upcoming satellite missions and their wide-field coverage in many frequencies also raise the possibility that non-Gaussian signals in the CMB temperature fluctuations may be experimentally detected and studied in detail. The deviations from Gaussianity in CMB temperature fluctuations arise through two possibilities: the existence of a primordial non-Gaussianity due to initial conditions [5] and the creation of non-Gaussian fluctuations through either the imprint of nonlinear growth of structures or mode-coupling effects by secondary sources of

\footnotetext{
*Also at: Sherman Fairchild Senior Research Fellow, Theoretical Astrophysics, California Institute of Technology, Pasadena, CA 91125. Email address: asante@hyde.uchicago.edu

${ }^{1}$ http://map.nasa.gsfc.gov

${ }^{2}$ http://astro.estec.esa.nl/Planck/; also, ESA D/SCI(6)3.
}

temperature fluctuations [6]. Note that in currently favored adiabatic CDM models, the primary non-Gaussian contribution is insignificant [5] and that main contributions in producing a non-Gaussian signal comes from nonlinear effects associated with large scale structure contributions to the $\mathrm{CMB}$ and through various mode coupling effects such as gravitational lensing.

As discussed in these papers, the detection of such nonGaussian signals at the three-point level is important for understanding inflation or large scale structure clustering. The direct detection of such non-Gaussian signals, however, through a measurement of the full angular bispectrum, the Fourier analog of the three-point correlation function, may be challenging. Similar to measurements of the bispectrum in the Cosmic Background Explorer (COBE) [7], it is likely that the future measurements will only be limited to certain configurations of the bispectrum, such as equilateral triangles in multipole space.

Keeping the possibility for experimental detections of the non-Gaussian signals in mind, we considered alternative statistics that include information from the higher order level but which can be extracted essentially through a modified two-point correlation function. In the present paper, we discuss the angular power spectrum associated with correlations between squared temperature and temperature. ${ }^{3}$ This power spectrum is essentially a compressed form of the bispectrum and can be computed using the same techniques that are well known with the measurements of the usual temperature an-

\footnotetext{
${ }^{3}$ Additionally, the squared temperature-squared temperature power spectrum can be used as a compressed measurement of the trispectrum. In Ref. [8], this was discussed as a possibility to extract the nonlinear kinetic Sunyaev-Zel'dovich effect, while in Ref. [9] this was considered as a possibility for extracting the lensing signal in CMB data.
} 
isotropy power spectrum. Thus we do not expect the measurement of the proposed statistic to be affected by issues related to computation as in the case of the full bispectrum.

As an illustration of the astrophysical uses of the squared temperature-temperature power spectrum, we consider the observational extraction of the bispectrum formed by nonlinear mode coupling due to gravitational lensing angular deflections in CMB data. Here we explicitly consider the correlation between lensing deflections and the SunyaevZel'dovich (SZ) effect [2] due to inverse-Compton scattering of CMB photons via hot electrons. Due to the spectral dependence of the SZ effect when compared to CMB thermal fluctuations, the SZ signal can be extracted from CMB fluctuations with multifrequency data (see, Ref. [3]). Here we use such a frequency separation of CMB and SZ maps, in the case of a Planck mission, and consider the measurement of the $\mathrm{CMB}^{2}-\mathrm{SZ}$ power spectrum. We show that this power spectrum is directly proportional to the cross-correlation between SZ and lensing deflections. Thus, with a measurement of the $\mathrm{CMB}^{2}-\mathrm{SZ}$ power spectrum in the Planck data, or in any other multifrequency data set with reliable frequency separation capabilities, one can directly probe the cross power spectrum formed by dark matter, traced by lensing, and pressure, traced by the SZ effect. The proposed method is one of the few ways to obtain this information, which is important for proper understanding of clustering of pressure relative to dark matter.

Though we only discuss the $\mathrm{CMB}^{2}$-SZ power spectrum as an application of the squared temperature-temperature power spectrum, there are additional applications in both CMB and large scale structure studies. Instead of measuring nonGaussian statistics such as skewness or the third moment, the proposed method can also be easily implemented for weak lensing observations of the large scale structure using galaxy shear data and for cross-correlation purposes between various probes of large scale structure.

The layout of the paper is as follows. In Sec. II, we present a general calculation of the temperature ${ }^{2}$-temperature angular power spectrum and its relation to the angular bispectrum of temperature anisotropies. In Sec. III, we illustrate the proposed angular power spectrum through a calculation of the expected signal and noise for the non-Gaussian signal produced through correlations between lensing angular deflection SZ effect. We discuss our results in Sec. IV, and conclude with a summary.

\section{CALCULATIONAL METHOD}

The bispectrum $B_{l_{1} l_{2} l_{3}}$ is a spherical harmonic transform of the three-point correlation function just as the angular power spectrum $C_{l}$ is the transform of the two-point function. In terms of the multipole moments of the temperature fluctuation field $T(\hat{\mathbf{n}})$,

$$
a_{l m}=\int d \hat{\mathbf{n}} T(\hat{\mathbf{n}}) Y_{l}^{m} *(\hat{\mathbf{n}}),
$$

the two-point correlation function is given by

$$
\begin{aligned}
C(\hat{\mathbf{n}}, \hat{\mathbf{m}}) & \equiv\langle T(\hat{\mathbf{n}}) T(\hat{\mathbf{m}})\rangle \\
& =\sum_{l_{1} m_{1} l_{2} m_{2}}\left\langle a_{l_{1} m_{1}}^{*} a_{l_{2} m_{2}}\right\rangle Y_{l_{1}}^{m_{1}} *(\hat{\mathbf{n}}) Y_{l_{2}}^{m_{2}}(\hat{\mathbf{m}}) .
\end{aligned}
$$

Under the assumption that the temperature field is statistically isotropic, the correlation is independent of $m$,

$$
\left\langle a_{l_{1} m_{1}}^{*} a_{l_{2} m_{2}}\right\rangle=\delta_{l_{1} l_{2}}^{\mathrm{D}} \delta_{m_{1} m_{2}}^{\mathrm{D}} C_{l_{1}},
$$

and is called the angular power spectrum. Likewise the threepoint correlation function is given by.

$$
\begin{aligned}
B(\hat{\mathbf{n}}, \hat{\mathbf{m}}, \hat{\mathbf{l}}) & \equiv\langle T(\hat{\mathbf{n}}) T(\hat{\mathbf{m}}) T(\hat{\mathbf{l}})\rangle \\
& \equiv \sum\left\langle a_{l_{1} m_{1}} a_{l_{2} m_{2}} a_{l_{3} m_{3}}\right\rangle Y_{l_{1}}^{m_{1}}(\hat{\mathbf{n}}) Y_{l_{2}}^{m_{2}}(\hat{\mathbf{m}}) Y_{l_{3}}^{m_{3}}(\hat{\mathbf{I}}),
\end{aligned}
$$

where the sum is over $\left(l_{1}, m_{1}\right),\left(l_{2}, m_{2}\right)$, and $\left(l_{3}, m_{3}\right)$. Statistical isotropy again allows us to express the correlation in terms of an $m$-independent function:

$$
\left\langle a_{l_{1} m_{1}} a_{l_{2} m_{2}} a_{l_{3} m_{3}}\right\rangle=\left(\begin{array}{ccc}
l_{1} & l_{2} & l_{3} \\
m_{1} & m_{2} & m_{3}
\end{array}\right) B_{l_{1} l_{2} l_{3}} .
$$

Here the quantity in parentheses is the Wigner- $3 j$ symbol. The orthonormality relation for Wigner- $3 j$ symbol implies

$$
B_{l_{1} l_{2} l_{3}}=\sum_{m_{1} m_{2} m_{3}}\left(\begin{array}{ccc}
l_{1} & l_{2} & l_{3} \\
m_{1} & m_{2} & m_{3}
\end{array}\right)\left\langle a_{l_{1} m_{1}} a_{l_{2} m_{2}} a_{l_{3} m_{3}}\right\rangle .
$$

The angular bispectrum $B_{l_{1} l_{2} l_{3}}$ contains all the information available in the three-point correlation function. For example, the skewness, the pseudocollapsed three-point function of Ref. [10] and the equilateral configuration statistic of Ref. [7] can all be expressed as linear combinations of the bispectrum terms (see Ref. [11] for explicit expressions, and Ref. [3] for an expression relating skewness in terms of the bispectrum).

The quantity of interest here is the correlation between squared temperature and temperature, instead of the usual temperature-temperature correlation. Following Eq. (2), we can write this power spectrum as

$$
\begin{aligned}
C^{2}(\hat{\mathbf{n}}, \hat{\mathbf{m}}) & \equiv\left\langle T^{2}(\hat{\mathbf{n}}) T(\hat{\mathbf{m}})\right\rangle \\
& =\sum_{l_{1} m_{1} l_{2} m_{2}}\left\langle a_{l_{1} m_{1}}^{2} a_{l_{2} m_{2}}^{*}\right\rangle Y_{l_{1}}^{m_{1}}(\hat{\mathbf{n}}) Y_{l_{2}}^{m_{2} *}(\hat{\mathbf{m}}) .
\end{aligned}
$$

Here, note that $a_{l_{1} m_{1}}^{2}$ is the multipole moments of the squared temperature field and not the square of the multipole moment of the temperature field, $\left(a_{l_{1} m_{1}}\right)^{2}$. Through the expansion of the temperature

$$
T(\hat{\mathbf{n}})=\sum a_{l m} Y_{l}^{m}(\hat{\mathbf{n}}),
$$


we can write

$$
\begin{aligned}
a_{l m}^{2} & =\int d \hat{\mathbf{n}} T^{2}(\hat{\mathbf{n}}) Y_{l}^{m} *(\hat{\mathbf{n}}) \\
& =\sum_{l_{1} m_{1} l_{2} m_{2}} a_{l_{1} m_{1}} a_{l_{2} m_{2}} \int d \hat{\mathbf{n}} Y_{l}^{m} *(\hat{\mathbf{n}}) Y_{l_{1}}^{m_{1}}(\hat{\mathbf{n}}) Y_{l_{2}}^{m_{2}}(\hat{\mathbf{n}})
\end{aligned}
$$

We can now construct the power spectrum of squared temperature and temperature as

$$
\begin{aligned}
\left\langle a_{l m}^{2} a_{l^{\prime} m^{\prime}}^{*}\right\rangle= & C_{l}^{2} \delta_{l l^{\prime}}^{\mathrm{D}} \delta_{m m^{\prime}}^{\mathrm{D}} \\
= & \sum_{l_{1} m_{1} l_{2} m_{2}}\left\langle a_{l_{1} m_{1}} a_{l_{2} m_{2}} a_{l^{\prime} m^{\prime}}^{*}\right\rangle \\
& \times \int d \hat{\mathbf{n}} Y_{l}^{m} *(\hat{\mathbf{n}}) Y_{l_{1}}^{m_{1}}(\hat{\mathbf{n}}) Y_{l_{2}}^{m_{2}}(\hat{\mathbf{n}}) .
\end{aligned}
$$

Using the Gaunt integral

$$
\begin{aligned}
\int \hat{\mathbf{n}} Y_{l_{1}}^{m_{1}} Y_{l_{2}}^{m_{2}} Y_{l_{3}}^{m_{3}}= & \sqrt{\frac{\left(2 l_{1}+1\right)\left(2 l_{2}+1\right)\left(2 l_{3}+1\right)}{4 \pi}} \\
& \times\left(\begin{array}{ccc}
l_{1} & l_{2} & l_{3} \\
0 & 0 & 0
\end{array}\right)\left(\begin{array}{ccc}
l_{1} & l_{2} & l_{3} \\
m_{1} & m_{2} & m_{3}
\end{array}\right),
\end{aligned}
$$

and introducing the bispectrum from Eq. (5), we can write the angular power spectrum of squared temperature and temperature as

$$
C_{l}^{2}=\sum_{l_{1} l_{2}} B_{l_{1} l_{2} l} w_{l_{1}, l_{2}}\left(\begin{array}{ccc}
l_{1} & l_{2} & l \\
0 & 0 & 0
\end{array}\right) \sqrt{\frac{\left(2 l_{1}+1\right)\left(2 l_{2}+1\right)}{4 \pi(2 l+1)}} .
$$

In simplifying, we have made use of the fact that

$$
\sum_{m_{1} m_{2}}\left(\begin{array}{ccc}
l_{1} & l_{2} & l \\
m_{1} & m_{2} & m
\end{array}\right)\left(\begin{array}{ccc}
l_{1} & l_{2} & l^{\prime} \\
m_{1} & m_{2} & m^{\prime}
\end{array}\right)=\frac{\delta_{l l^{\prime}}^{\mathrm{D}} \delta_{m m^{\prime}}^{\mathrm{D}}}{2 l+1} .
$$

Equation (12) is the expression of interest here. This relates the angular bispectrum to the angular power spectrum of squared temperature and temperature. In taking a general approach, we have introduced a filter, or window, function in Fourier space of $w_{l_{1}, l_{2}}$. Later we will discuss detailed forms for the appropriate filter functions later.

Since the bispectrum is defined by a triangle in multipole space with lengths of sides $\left(l, l_{1}, l_{2}\right)$, the $C_{l}^{2}$ power spectrum essentially captures information, through a summation, associated with all triangular configurations with one of the sides of length $l$. If it is known a priori that certain triangular configurations contribute to the bispectrum significantly, such as flattened triangles in the case of certain secondary correlations (see, Ref. [6]), one can compute this sum by only restricting the multipoles of interest. This is essentially what can be achieved with the introduction of an appropriate window, or a filter, function to the squared temperature field. Though the expression for the angular power spectrum involves a bispectrum, the experimental measurement is straightforward: one constructs the power spectrum by squaring the temperature field, in real space, and using the Fourier transforms of squared temperature values and the temperature field, with any filtering functions, when necessary.

We will now discuss the measurement of the power spectrum using Planck data for the non-Gaussian signal involved through the correlation between lensing angular deflections and the SZ effect. This bispectrum has the high cumulative signal-to-noise ratio out of all other possible bispectra involving secondary effects and CMB temperature [6]. For the illustration of our results we use the currently favored $\Lambda$ CDM cosmological model. The parameters for this model are $\Omega_{c}=0.30, \Omega_{b}=0.05, \Omega_{\Lambda}=0.65, h=0.65, Y_{p}=0.24, n$ $=1$, and COBE normalization $\delta_{H}=4.2 \times 10^{-5}$ [12]. This model has mass fluctuations on the $8 \mathrm{~h} \mathrm{Mpc}^{-1}$ scale in accord with the abundance of galaxy clusters $\sigma_{8}=0.86$ [13].

\section{CMB ${ }^{2}$-SZ POWER SPECTRUM}

We refer the reader to Ref. [6] for a full derivation of the bispectra due to correlations between lensing angular deflections in the CMB and secondary effects due to large scale structure. Following these derivations, we can write the bispectrum as

$$
\begin{aligned}
B_{l_{1}, l_{2}, l}= & -\left(\begin{array}{ccc}
l_{1} & l_{2} & l \\
0 & 0 & 0
\end{array}\right) \sqrt{\frac{\left(2 l_{1}+1\right)\left(2 l_{2}+1\right)(2 l+1)}{4 \pi}} \\
& \times\left[f_{l_{2}, l_{1}, l} C_{l_{1}}^{\mathrm{CMB}} b_{l}+\text { Perm. }\right]
\end{aligned}
$$

where

$$
f_{l_{a}, l_{b}, l_{c}}=\left[\frac{l_{a}\left(l_{a}+1\right)-l_{b}\left(l_{b}+1\right)-l_{c}\left(l_{c}+1\right)}{2}\right],
$$

and $b_{l}$ is the cross-correlation power spectrum involved with lensing angular deflections and a secondary source of anisotropies that trace the large scale structure and effectively correlates with lensing potentials (e.g., the integrated Sachs-Wolfe effect [14], the linear Doppler effect, and the $\mathrm{SZ}$ effect). Here we consider the case of SZ since, as discussed in Ref. [6], the correlation between lensing and other two effects does not lead to a bispectrum with a significant signal-to-noise ratio.

If the two maps involved with the construction of the squared temperature-temperature power spectrum is the same (e.g., CMB alone), then the permutation in Eq. (14) leads to a total of six terms in the ordering of $l_{1}, l_{2}$, and $l[6]$. When the bispectrum is measured with two independent maps, e.g., $\mathrm{CMB}$ and SZ through frequency cleaning, the permutation in Eq. (14) involves an additional term with the replacement of $l_{1}$ by $l_{2}$.

We now primarily consider the case of two independent maps with Planck data, and thus, we can write the $\mathrm{CMB}^{2}-\mathrm{SZ}$ power spectrum as 


$$
\begin{aligned}
C_{l}^{2}= & b_{l} \sum_{l_{1} l_{2}}\left(\begin{array}{ccc}
l_{1} & l_{2} & l \\
0 & 0 & 0
\end{array}\right)^{2} \frac{\left(2 l_{1}+1\right)\left(2 l_{2}+1\right)}{4 \pi} w_{l_{1}, l_{2}}^{2} \\
& \times\left[C_{l_{1}}^{\mathrm{CMB}} f_{l_{2}, l_{1}, l}+C_{l_{2}}^{\mathrm{CMB}} f_{l_{1}, l_{2}, l}\right] .
\end{aligned}
$$

Here $C_{l}^{\mathrm{CMB}}$ is the unlensed primary CMB contribution.

The proposed use of the $\mathrm{CMB}^{2}-\mathrm{SZ}$ power spectrum is essentially the following. One can directly measure $C_{l}^{2}$ in the CMB and SZ data and since information related to $C_{l}^{\mathrm{CMB}}$ also directly comes from data, one can construct the quantity $b_{l}$ which is directly proportional to $C_{l}^{2}$. As written in Eq. (16), in the case relevant to the $\mathrm{CMB}^{2}-\mathrm{SZ}$ power spectrum, the cross-correlation between lensing deflections and the SZ effect:

$$
b_{l}=\int d r \frac{\left.W^{\mathrm{SZ}}(r) W^{\mathrm{len}}(k, r)\right|_{k=l / d_{A}}}{d_{A}^{2}} P_{\Pi \delta}\left(\frac{l}{d_{A}} ; r\right) .
$$

Here we have utilized the Limber approximation [15] by setting $k=l / d_{A}, P_{\Pi \delta}$ is the pressure-dark matter cross power spectrum, $r$ is the comoving radial distance, and $d_{A}$ is the comoving angular diameter distance. The window functions for the SZ and lensing deflections are

$$
W^{\mathrm{SZ}}(r)=g(x) \frac{k_{B} \sigma_{T} \bar{n}_{e}}{a(r)^{2} m_{e} c^{2}}
$$

and

$$
W^{\operatorname{len}}(k, r)=-3 \frac{\Omega_{m}}{a(r)}\left(\frac{H_{0}}{k}\right)^{2} \frac{d_{A}\left(r_{s}-r\right)}{d_{A}(r) d_{A}\left(r_{s}\right)},
$$

respectively. In Eq. (18), $\bar{n}_{e}$ is the mean density of electrons today, $\sigma_{T}$ is the Thomson cross section and $g(x)$ $=x \operatorname{coth}(x / 2)-4$ with $x=h \nu / k_{B} T_{\mathrm{CMB}}$ is the spectral shape of the SZ effect. At Rayleigh-Jeans part of the CMB, $g(x)$ $=-2$. As discussed in [3], an experiment such as that of Planck, with observations over a wide range of frequencies, can separate out SZ contributions based on the spectral signature, $g(x)$ relative to primary $\mathrm{CMB}$ and other contributors. For this paper, we will consider Planck data in estimating the signal-to-noise ratio, and will use the noise power spectra calculated in Ref. [3] for the Planck SZ and CMB maps.

In order to assess how well Planck CMB and SZ maps can be used for the purpose of constructing $b_{l}$, we need to consider an estimate for it, as well as estimates for $C_{l}^{\mathrm{CMB}}$ and $C_{l}^{\mathrm{SZ}}$. The latter is required when estimating the signal-tonoise ratio for possible detections. We use CMBFAST of Ref. [16] for $C_{l}^{\mathrm{CMB}}$ and compute $b_{l}$ and $C_{l}^{\mathrm{SZ}}$ analytically using a semianalytical description of pressure fluctuations in the large scale structure involving a halo approach [17]. To calculate $b_{l}$ we need the pressure-dark matter cross-power spectrum, while $C_{l}^{\mathrm{SZ}}$ depends on the pressure-pressure power spectrum. The power spectra are introduced and discussed in detail in Refs. [18] and [8], and we refer the reader to these two papers for further details on this approach. The basic assumption of the halo model is that large scale structure

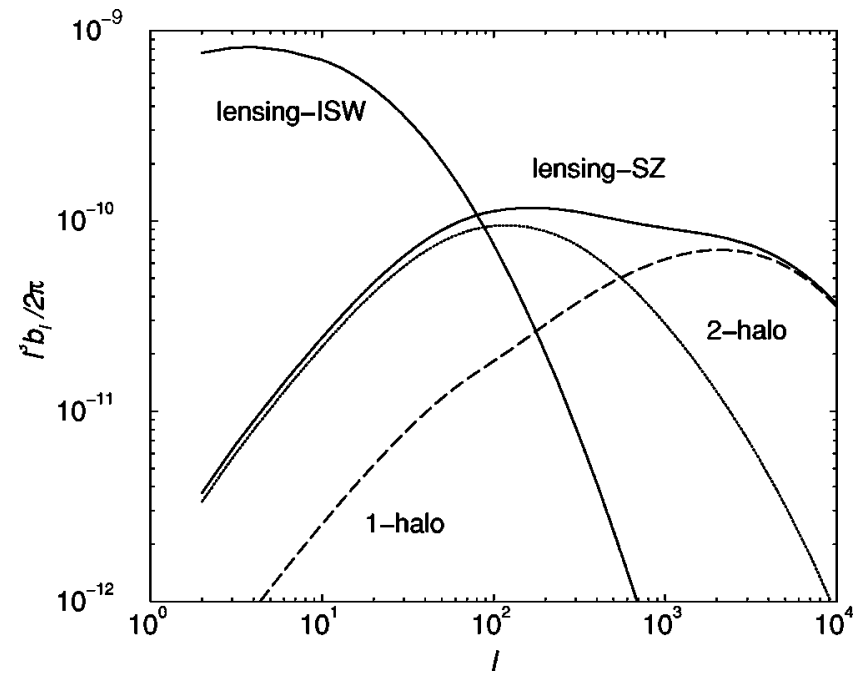

FIG. 1. Power spectrum for the correlation of lensing angular deflections and the SZ effect, as calculated under the halo description. As shown, most of the contributions to the lensing-SZ correlation, as relevant for CMB, comes from large angular scale correlations between halos, denoted by the two-halo term, and the mildly nonlinear regime where contributions from individual halos are important (one-halo term). For reference, we also show the correlation power spectrum for lensing deflections and the integrated SachsWolfe effect at late times.

dark matter distribution can be viewed as a collection of dark matter halos with a mass function following Press-Schechter [19] mass functions or variants and a dark matter distribution in each halo following NFW profile of Ref. [20] or other descriptions. The halos are biased relative to the linear density field following the description of Ref. [21]. With clustering of dark matter described through such an approach, any other physical property of the large scale structure can be easily described through the relation between that property and dark matter. For example, in the case of pressure, the gas distribution in each halo follows hydrostatic equillibrium with dark matter. As discussed in Ref. [8], such an approach through the halo model provides a reliable semianalytical approach to model nonlinear large scale structure clustering and its predictions, in the case of pressure as relevant for the SZ effect and lensing SZ correlations, are consistent with numerical simulations (e.g., Ref. [22]).

In Fig. 1, we show the correlation between lensing deflections and the SZ effect. The contributions are broken to single one-halo, and two-halo terms involving correlations between and within halos. As shown, for multipoles of interest, most of the correlation comes from the two-halo term involving large scale correlations between halos, though at multipoles asymptotically equal to a few hundred, the contribution from a single halo term cannot be ignored. This is the mildly nonlinear regime where contributions from large scale correlations between halos and the halo profiles become important.

Following the correlation between lensing and SZ shown in Fig. 1, in Fig. 2, we show the $C_{l}^{2}$ power spectrum due to the relevant bispectrum. We now discuss the calculation of errors shown in Fig. 2. 


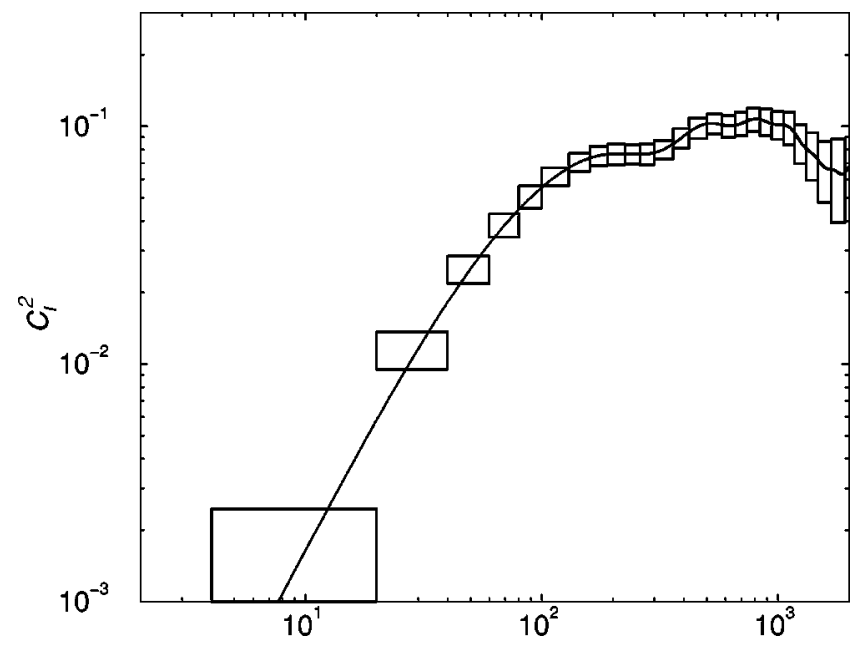

FIG. 2. The angular power spectrum of $\mathrm{CMB}^{2}-\mathrm{SZ}$ due to the correlation of the SZ effect and gravitational lensing in the CMB. The band power errors are for the Planck mission using the noise calculation for multifrequency separation of SZ and CMB effects from Planck data.

\section{A. Signal-to-noise ratio}

To establish the signal-to-noise ratio for the detection of the lensing-SZ bispectrum through the squared temperaturetemperature power spectrum, we calculate the covariance associated with the proposed statistic following Ref. [8]. Here we assume that the squared temperature and temperature power spectrum will be measured with two maps involving $\mathrm{CMB}$ and SZ data with a squared temperature measurement from the $\mathrm{CMB}$ map and a single temperature measurement from SZ. We can write the error on each of the power spectrum measurements associated with the proposed statistic as

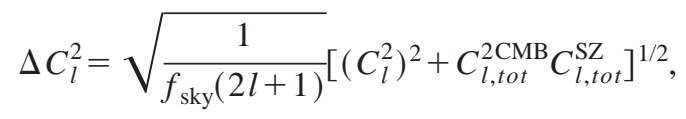

while the total signal-to-noise ratio is

$$
\frac{S}{N}=\left[f_{\text {sky }} \sum_{l}(2 l+1) \frac{\left(C_{l}^{2}\right)^{2}}{\left(C_{l}^{2}\right)^{2}+C_{l, t o t}^{2 \mathrm{CMB}} C_{l, t o t}^{\mathrm{SZ}}}\right]^{1 / 2},
$$

with the fraction of sky covered by $f_{\text {sky }}$.

Here the proposed squared temperature and temperature angular power spectrum is $C_{l}^{2}$, and $C_{l, t o t}^{2 \mathrm{CMB}}$ is the squared temperature power spectrum in $\mathrm{CMB}$ data alone:

$$
\begin{aligned}
C_{l, t o t}^{2 \mathrm{CMB}}= & \sum_{l_{1} l_{2}} 2 C_{l_{1}, t o t}^{\mathrm{CMB}} C_{l_{2}, t o t}^{\mathrm{CMB}} w_{l_{1}, l_{2}}^{2} \\
& \times\left(\begin{array}{ccc}
l_{1} & l_{2} & l_{3} \\
0 & 0 & 0
\end{array}\right)^{2} \frac{\left(2 l_{1}+1\right)\left(2 l_{2}+1\right)}{4 \pi} .
\end{aligned}
$$

In calculating the noise, following Ref. [23], we also include relevant detector and frequency separation noise-for the SZ map-by introducing $C_{l, t o t}^{\mathrm{CMB}}=C_{l}^{\mathrm{CMB}}+C_{l}^{\mathrm{S}}+C_{l}^{\mathrm{N}}$, with $S$ denoting other secondary contributors in the CMB map (e.g., lensing), and $N$ is the noise contribution. There is an additional term here involving the $\mathrm{CMB}$ trispectrum which leads to the covariance between multipoles. We ignore this here, given that there is no significant non-Gaussian component to the primary anisotropies especially in the adiabatic CDM models [5], which are more consistent with current observations of large scale structure and CMB. The next important contribution to the covariance of the CMB comes from secondary effects with a thermal spectrum. As discussed by $\mathrm{Hu}$ [9], gravitational lensing of the $\mathrm{CMB}$, which is the most important secondary effect that leads to nonGaussian correlations, does not produce a significant covariance out to $l$ of 2000 as relevant for the Planck mission. Thus we ignore the presence of a non-Gaussian trispectrum in our variance estimates and only consider Gaussian contributions. At further small angular scales, in addition to lensing, other secondary effects with a CMB thermal spectral signature, such as two kinetic SZ effect, can produce a trispectrum in CMB temperature data (see, Ref. [8]).

Note that since we consider frequency separated maps, there is no trispectrum contribution to the covariance of the proposed statistic from the SZ effect and contribution to the variance comes only from the $\mathrm{SZ}$ power spectrum $C_{l}^{\mathrm{SZ}}$. We calculated this power spectrum again using the halo model following [8]. If the measurement is to be done in a map which is not frequency cleaned with components separated out, then the trispectrum involved with the SZ model will also contribute to the covariance.

\section{B. Filter functions}

Here we consider two possibilities for the filtering of unnecessary noise in the $T^{2}$ map. A straightforward filter is to essentially remove the excess low multipole noise and we achieve this by choosing

$$
w_{l_{1}, l_{2}}= \begin{cases}1, & l_{1}, l_{2}>l_{\mathrm{cut}} \\ 0, & l_{1}, l_{2}<l_{\mathrm{cut}}\end{cases}
$$

where we set the $l_{\text {cut }}$ to be 1000 . Note that in the case of Planck, instrumental noise degrades the information beyond a value of $l$ equal to 2000 .

Additionally, we consider an optimal filtering scheme which maximizes the $(S / N)^{2}$ in Eq. (21). Analytically, this is equivalent to taking the derivative of the expression in Eq. (21) with respect to $w_{l_{1}, l_{2}}$ and solving for the value which maximizes the signal-to-noise ratio. We found this to occur when

$$
w_{l_{1}, l_{2}}=\frac{\left[C_{l_{1}}^{\mathrm{CMB}} f_{l_{2}, l_{1}, l}+C_{l_{2}}^{\mathrm{CMB}} f_{l_{1}, l_{2}, l}\right]}{2 C_{l, t o t}^{\mathrm{CMB}} C_{l_{2}, \text { tot }}^{\mathrm{CMB}}}
$$

with an appropriate normalization such that $\Sigma_{l_{1} l_{2}} w_{l_{1}, l_{2}}=1$. This optimal filter is equivalent to the one introduced by $\mathrm{Hu}$ [9] for the estimator of the deflection angles in the CMB using a quadratic statistic. Note that the optimal filter is independent of the correlation power between lensing deflections and the SZ effect; however, it depends on the details of mode coupling associated with lensing in the CMB through $f$ 
terms. One can easily construct the filter through a priori knowledge on the CMB power spectrum and noise properties.

When the optimal filter is applied to the squared temperature map, $C_{l, t o t}^{2 \mathrm{CMB}}=C_{l}^{2}$, this leads to a simplified expression for the signal-to-noise ratio of the $\mathrm{CMB}^{2}-\mathrm{SZ}$ power spectrum:

$$
\begin{aligned}
\left(\frac{S}{N}\right)^{2} \approx & f_{\text {sky }} \sum_{l l_{1} l_{2}}\left(\begin{array}{ccc}
l_{1} & l_{2} & l \\
0 & 0 & 0
\end{array}\right)^{2} \frac{\left(2 l_{1}+1\right)\left(2 l_{2}+1\right)}{4 \pi} \\
& \times \frac{\left[C_{l_{1}}^{\mathrm{CMB}} f_{l_{2}, l_{1}, l}+C_{l_{2}}^{\mathrm{CMB}} f_{l_{1}, l_{2}, l}\right]^{2} b_{l}^{2}}{2 C_{l_{1}, t o t}^{\mathrm{CMB}} C_{l_{2}, t o t}^{\mathrm{CMB}} C_{l, t o t}^{\mathrm{SZ}}} \\
= & f_{\text {sky }} \sum_{l l_{1} l_{2}} \frac{B_{l_{1} l_{2} l}^{2}}{2 C_{l_{1}, t o t}^{\mathrm{CMB}} C_{l_{2}, t o t}^{\mathrm{CMB}} C_{l, t o t}^{\mathrm{SZ}}} .
\end{aligned}
$$

As written above, the total signal-to-noise ratio is now equal to exactly the total amount present in the full bispectrum formed by correlations between gravitational lensing deflections in CMB and SZ effects (see, Ref. [6]). Thus the optimal filter allows one to capture, at the two point level through basically a power spectrum, all information contained within the bispectrum at the three point level; this happens with no loss in the signal-to-noise ratio. The temperature-gradient divergence statistic introduced in Ref. [9] allows this to be carried out directly; with appropriate optimal filtering suggested above, this is equivalent to the $\mathrm{CMB}^{2}$-SZ approached outlined here.

In Fig. 3, we show the surface of the optimal filter as a function of $l_{1}$ and $l_{2}$ when $l_{3}=100$ and 2000, respectively. As shown, the optimal filter behaves such that one only used information in multipole range with $l_{1}$ and $l_{2} \gtrsim 1500$. This behavior is the reason why a simple filter with the behavior of a step function in $l_{1}$ and $l_{2}$ essentially allows us to capture some of the information present in the bispectrum. The detailed behavior of the optimal filter function, especially information related to lensing present in the peaks and valleys of the CMB power spectrum, however, limits the information that can be captured by a simple filter. The optimal filter behaves such that it effectively extracts all information related to lensing from the damping tail of the CMB power spectrum.

\section{DISCUSSION}

In Fig. 1, we show the correlation between SZ effect and lensing angular deflections. The cross angular power spectrum is weighted by a factor of $l^{3}$ to highlight the multipole range which is important for the CMB bispectrum due to gravitational lensing-secondary correlations. For comparison, in the same plot, we show the correlation power between lensing deflections and the integrated Sachs-Wolfe (ISW) effect [14] at late times. We refer the reader to Cooray and $\mathrm{Hu}$ [6] for a detailed discussion of the bispectra produced through correlations between gravitational lensing angular deflections and secondary effects. In the case of the
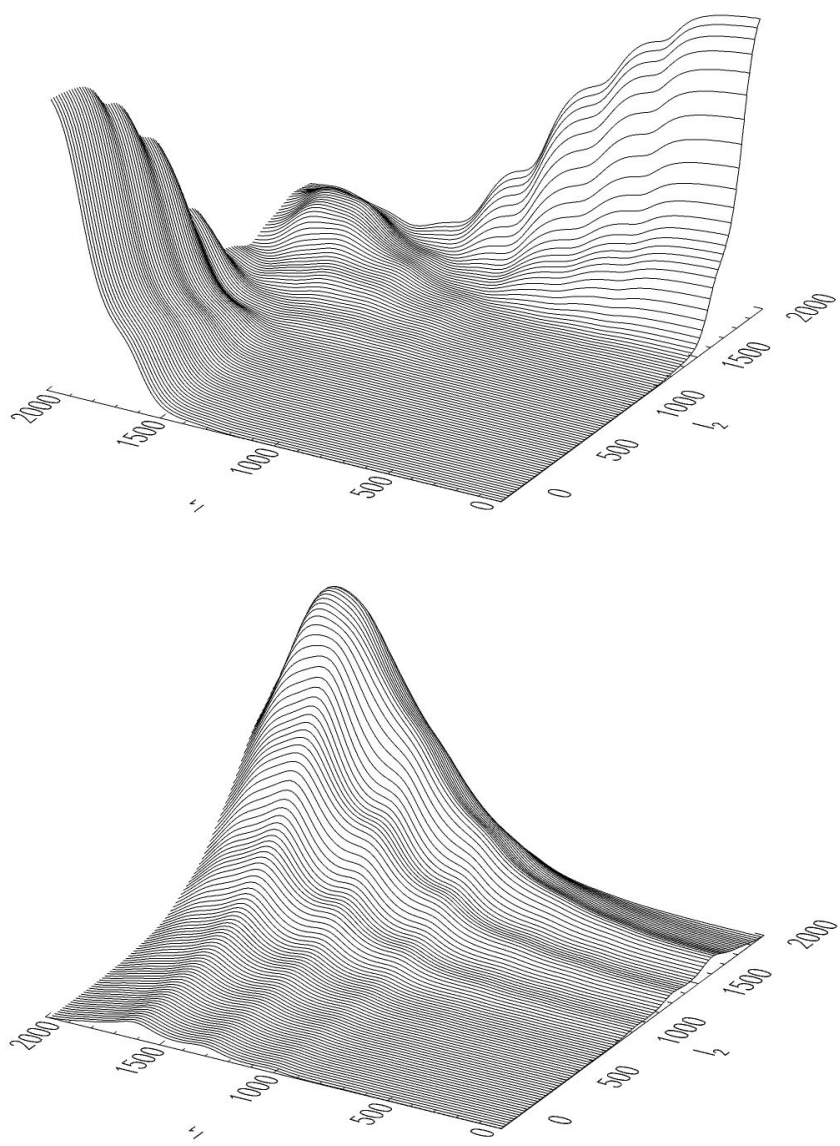

FIG. 3. Optimal filter function for the $\mathrm{CMB}^{2}-\mathrm{SZ}$ power spectrum, as a function of $l_{1}$ and $l_{2}$. In left, the filter is when $l=100$ and right is when $l=2000$ corresponding to large and small angular scales respectively. The optimal filter behaves such that it removes excess noise at multipoles less than $\sim 1500$, and extracts sensitivity of lensing in the damping tail of the power spectrum.

lensing-SZ effect, we note that we have updated the calculations presented therein using a detailed description of large scale structure pressure fluctuations instead of the assumption that pressure traces dark matter (see, Refs. $[18,8]$ for a discussion on the computation of statistics related to large scale structure pressure). The assumption of pressure traces dark matter, in general, leads to a slight overestimate of the correlation between lensing and the SZ effect.

The lensing-SZ correlation behaves such that contributions relevant to the CMB bispectrum, at multipoles less than 100 , comes from interhalo large scale correlations, which we defined as the two-halo term in Ref. $[18,8]$, involving the linear density field. The mildly nonlinear regime, at multipoles around a few hundred, is described through the combination of large scale correlations and the interhalo correlations through the profiles denoted by the one-halo term. This behavior is consistent with the fact that angular deflections in the CMB is a large scale phenomena with an angular coherence of the deflection angle of $\sim 10^{\circ}$ (see Ref. [24]). Though lensing window function peaks at a redshift of 3 , at half the angular diameter distance to the last scattering (recombination), the nonlinear contributions to the lensing-SZ correlations comes primarily from the SZ effect, which has a win- 
dow function that effectively peaks at very low redshifts; overall, contributions to the lensing-SZ correlations comes over a wide range of redshifts and the low redshift behavior of the SZ effect makes the contribution from the one-halo term important at small angular scales.

Note that most of the contributions come at multipoles greater than a few hundred, while a tail continues to multipoles of thousands. Since the CMB itself dominates low multipoles, its contribution to the variance is more important at multipoles less than $\sim 1000$. This is the same reason why the lensing-ISW effect, even with a significantly higher correlation at very large angular scales, leads to a considerably smaller signal-to-noise ratio for the bispectrum, and thus to the squared temperature-temperature power spectrum.

In Fig. 2, we show the angular power spectrum of $\mathrm{CMB}^{2}$-SZ power spectrum using the halo model description of $b_{l}$. We computed the power spectrum band errors by using the optimal filtering scheme discussed in Sec. III B and shown in Fig. 3, and using Eq. (20) to compute errors. Here we consider the Planck measurement of the SZ effect, and use the variance power spectrum calculated in Ref. [3]. We also include the relevant beam and detector noise in the CMB map. For secondary contributors to the noise, we include the contribution from lensing and nonlinear kinetic SZ effects following Refs. [24] and [8]. The effect of the optimal filter is to remove the noise associated with low multipoles primarily from the intrinsic $\mathrm{CMB}$ anisotropies, while extracting information related to lensing associated with peaks and valleys in the CMB power spectrum as well as small scale information coming from the modification of a CMB gradient on the sky. As shown, Planck derived CMB and SZ maps can be clearly used to detect the $\mathrm{CMB}^{2}$-SZ non-Gaussian bispectrum due to lensing at the two-point level.

In Fig. 4, we show the cumulative signal-to-noise ratio associated with this detection. Here we consider both the optimal filtering as well as our crude filter where we removed all information at multipoles less than 1000. In the top panel, we consider this latter scenario. Using the frequency cleaned maps, the signal-to-noise ratio ranges up to $\sim 20$, while with no frequency cleaning we only find a value around 6. If Planck were to allow perfect separation of SZ and $\mathrm{CMB}$ and with no noise contributions to both SZ and CMB maps, and with only cosmic variance, one finds a total signal-to-noise ratio of $\sim 60$. This increase in the signal-tonoise ratio primarily comes from multipoles greater than 1000 , and is due to the decrease in the noise associated with the SZ map. Clearly, improving the separation of the SZ effect from the $\mathrm{CMB}$, and decreasing the associated noise, can lead to a significantly better detection of the lensing-SZ correlation through the $\mathrm{CMB}^{2}$-SZ power spectrum.

In the bottom panel of Fig. 4, we compare cumulative signal-to-noise ratio with no filtering, optimal filtering, and with our simple noise removal at $l \lesssim 1000$ and $l \lesssim 1500$. Here again, we consider the Planck CMB and SZ maps. With no filtering, the cumulative signal-to-noise ratio is of order $\sim 3$, while with optimal filtering, one obtains a total signal-tonoise ratio of $\sim 70$. With the simple noise removal at $l$ of 1000 , one has only gained a factor of $\sim 6$, while this is still a factor of $\sim 3.5$ less than optimal case. As shown in Fig. 3,
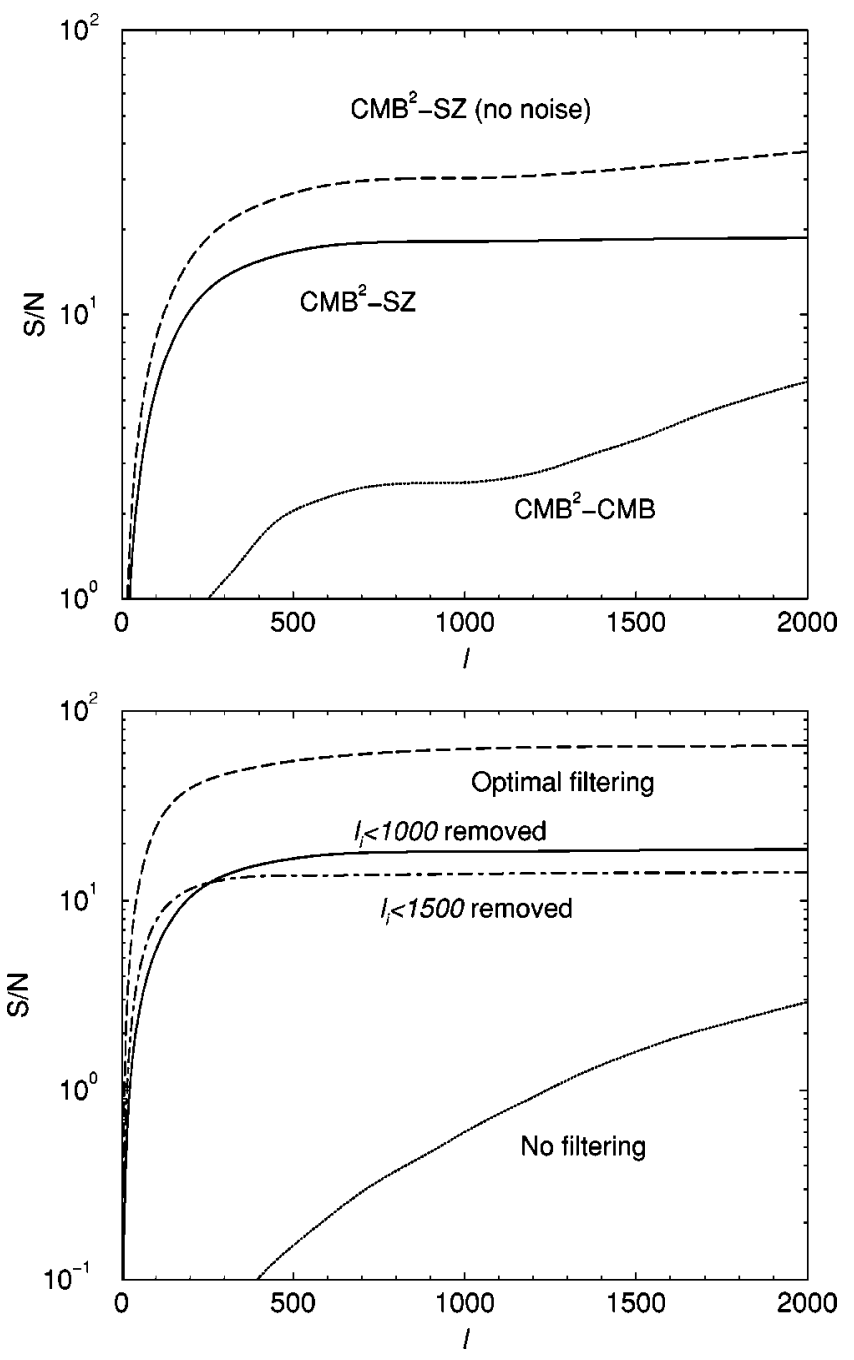

FIG. 4. The cumulative signal-to-noise ratio for the detection of temperature ${ }^{2}$-temperature power spectra in Planck data. The top figure uses the filtering scheme where we remove excess noise at multipoles $l_{1}$ and $l_{2}$ of less than 1000. The shown curves are for with no SZ separation (dotted curve), with SZ separation (solid curve), and perfect SZ separation with no noise contribution to both $\mathrm{SZ}$ and CMB maps (dashed curve). In the realistic case of SZ separation, the total cumulative signal-to-noise ratio is of order $\sim 20$. The bottom panel compares the signal-to-noise for Planck with no filtering (dotted line) and optimal filtering (long dashed line). Optimal filtering leads to a cumulative signal-to-noise ratio of $\sim 70$.

when large scales are considered, the filter removes excess noise out to $l_{1}$ and $l_{2}$ of 1500 . Thus we introduced simple noise removal out to $l$ of 1500. As shown in Fig. 4 (bottom panel) by a dot-dashed line, even though there is an increase in the signal-to-noise ratio at low $l$ values, consistent with the behavior of the optimal filter function, one finds a less cumulative signal-to-noise ratio at small angular scales. Such differences are primarily due to the detailed behavior of the optimal filter. With a simple filter, no information is obtained from multipoles that is associated with peaks and valleys of the CMB power spectrum, while related information is contained within the optimal filter. Though one can obtain a 
relatively modest measurement of the $\mathrm{CMB}^{2}$-SZ power spectrum through crude filtering, an optimal filter, such as the one suggested here, is highly recommended to exploit the full potential of the measurable signal.

In addition to the $\mathrm{CMB}^{2}$-SZ power spectrum, or a constructing through the temperature-gradient divergence statistics of Ref. [9], an alternative technique to construct the lensing-SZ correlation involves the gradient statistic of Ref. [25]. This technique utilizes gradient maps and an analysis similar to polarization to construct a map that is directly proportional to weak lensing convergence. With such a statistic, and following the procedure in Refs. [25,26], we find a cumulative signal-to-noise ratio of $\sim 35$, which is better than the simple filter but roughly a factor of 2 worse than the optimal filter considered here.

Given that the proposed statistic involving $\mathrm{CMB}^{2}-\mathrm{SZ}$ power spectrum is directly proportional to the cross-power spectrum between lensing angular deflections and the SZ effect, our error estimates on $C_{l}^{2}$ can be converted to those of $b_{l}$. In Fig. 5, we show the band power errors on $b_{l}$ using the Planck estimates for errors on the $\mathrm{CMB}^{2}$-SZ power spectrum using the simple filter (dashed line) and the optimal filter (solid lines). As shown, the Planck estimate allows a clear detection of the cross-correlation between SZ and lensing deflections; the detection is very clear with the optimal filter. The experimental measurement of this correlation power spectrum is preferred since it contains important cosmological and astrophysical information related to large scale fluctuations of pressure and dark matter. When combined with a SZ-SZ power spectrum and a lensing-lensing power spectrum either from large scale structure weak lensing or the CMB directly, this lensing-SZ power spectrum will provide knowledge about the correlation between pressure and dark matter. The proposed squared temperature-temperature statis-

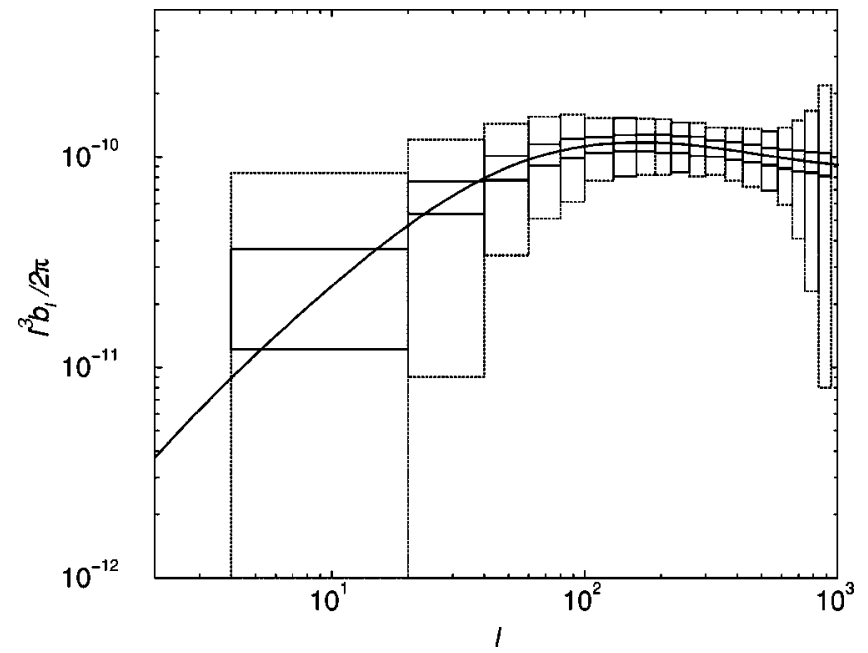

FIG. 5. Power spectrum for the correlation of lensing angular deflections and the SZ effect, as calculated under the halo description. The band power errors are from the measurement of the $\mathrm{CMB}^{2}$-SZ power spectrum in Planck with frequency cleaned CMB and SZ maps with a filtering scheme where noise is removed at multipoles less than 1000 (dotted) and using optimal filtering (solid). The experimental measurement of the SZ-lensing deflections cross power spectrum is important for understanding of relation between large scale pressure and dark matter fluctuations.

tic involving Planck CMB and SZ maps, with appropriate filtering, is clearly one of the few ways to obtain this information.

\section{ACKNOWLEDGMENTS}

We acknowledge the use of CMBFAST of U. Seljak and M. Zaldarriaga [16]. We thank Wayne Hu for many useful discussions.
[1] L. Knox, Phys. Rev. D 52, 4307 (1995); G. Jungman, M. Kamionkowski, A. Kosowsky, and D.N. Spergel, ibid. 54, 1332 (1996); J.R. Bond, G. Efstathiou, and M. Tegmark, Mon. Not. R. Astron. Soc. 291, L33 (1997); M. Zaldarriaga, D.N. Spergel, and U. Seljak, Astrophys. J. 488, 1 (1997); D.J. Eisenstein, W. Hu, and M. Tegmark, ibid. 518, 2 (1999).

[2] R.A. Sunyaev and Ya.B. Zel'dovich, Mon. Not. R. Astron. Soc. 190, 413 (1980).

[3] A. Cooray, W. Hu, and M. Tegmark, Astrophys. J. 540, 1 (2000).

[4] L. Knox, A. Cooray, D. Eisenstein, and Z. Haiman, Astrophys. J. 550, 7 (2001).

[5] E. Komatsu and D.N. Spergel, Phys. Rev. D 63, 063002 (2001); L. Wang and M. Kamionkowski, ibid. 61, 063504 (2000); A. Gangui and J. Martin, Mon. Not. R. Astron. Soc. 313, 323 (2000); X. Luo and D.N. Schramm, Phys. Rev. Lett. 71, 1124 (1994); X. Luo, Astrophys. J. Lett. 427, L71 (1994); T. Falk, R. Rangarajan, and M. Frednicki, ibid. 403, L1 (1993).

[6] A. Cooray and W. Hu, Astrophys. J. 534, 533 (2000); D.M. Goldberg and D.N. Spergel, Phys. Rev. D 59, 103002 (1999);
D.N. Spergel and D.M. Goldberg, ibid. 59, 103001 (1999).

[7] P.G. Ferreira, J. Magueijo, and K.M. Gorksi, Astrophys. J. 503, 1 (1998); for updates, also see, J. Pando, D. Valls-Gabaud, and L.Z. Fang, Phys. Rev. Lett. 81, 4568 (1998); A.J. Banday, S. Zaroubi, and K.M. Gorski, Astrophys. J. 533, 575 (2000); B. Bromley and M. Tegmark, Astrophys. J. Lett. 524, L79 (1999).

[8] A. Cooray, Phys. Rev. D (to be published), astro-ph/0105063; and Ph.D. thesis, University of Chicago, Chicago, 2001, available from the University of Chicago Crear Science Library or from the author.

[9] W. Hu, Phys. Rev. D. (to be published), astro-ph/0105117.

[10] G. Hinshaw, A.J. Banday, C.L. Bennett, K.M. Gorski, and A. Kogut, Astrophys. J. 446, 67 (1995).

[11] A. Gangui, F. Lucchin, S. Matarrese, and S. Mollerach, Astrophys. J. 430, 447 (1994).

[12] E.F. Bunn and M. White, Astrophys. J. 480, 6 (1997).

[13] P.T.P. Viana and A.R. Liddle, Mon. Not. R. Astron. Soc. 303, 535 (1999)

[14] R.K. Sachs and A.M. Wolfe, Astrophys. J. 147, 73 (1967).

[15] D. Limber, Astrophys. J. 119, 655 (1954). 
[16] U. Seljak and M. Zaldarriaga, Astrophys. J. 469, 437 (1996).

[17] R.J. Scherrer and E. Bertschinger, Astrophys. J. 381, 349 (1991); R.K. Sheth and B. Jain, Mon. Not. R. Astron. Soc. 285, 231 (1997); U. Seljak, ibid. 318, 203 (2000); C.-P. Ma and J.N. Fry, Astrophys. J. 543, 503 (2000); A. Cooray and W. Hu, ibid. 548, 7 (2001); R. Scoccimarro, R. Sheth, L. Hui, and B. Jain, ibid. 546, 20 (2001).

[18] A. Cooray, Phys. Rev. D 62, 103506 (2000).

[19] W.H. Press and P. Schechter, Astrophys. J. 187, 425 (1974); also see, R.K. Sheth and B. Tormen, Mon. Not. R. Astron. Soc. 308, 119 (1999).
[20] J. Navarro, C. Frenk, and S.D.M. White, Astrophys. J. 462, 563 (1996).

[21] H.J. Mo, Y.P. Jing, and S.D.M. White, Mon. Not. R. Astron. Soc. 284, 189 (1997); H.J. Mo and S.D.M. White, ibid. 282, 347 (1996).

[22] A. Refregier and R. Teyssier, astro-ph/0012086.

[23] L. Knox, Phys. Rev. D 52, 4307 (1995).

[24] W. Hu and A. Cooray, Phys. Rev. D 63, 023504 (2001).

[25] M. Zaldarriaga and U. Seljak, Phys. Rev. D 59, 123507 (1999).

[26] H.V. Peiris and D.N. Spergel, Astrophys. J. 540, 605 (2000). 